\title{
Genetic and imaging study of a case with multiple malformations: limb body wall complex
}

\begin{abstract}
The Limb-Wall Complex is a rare and sporadic congenital anomaly characterized by multiple craniofacial and thoracoabdominal malformations as well as involvement of the spine and lower extremities. The etiology is unknown and the chromosomal study is normal, however, it is related to an alteration in the formation of the umbilical cord (short umbilical cord), abdominal placental insertion and persistence of the extraembryonic coelom. This condition causes intracavitary organs to be exposed and adhered to the placenta and consequently also attached to the mother's uterus. The diagnosis can be made from the first trimester of gestation with a nuchal translucency ultrasound in order to detect this pathology in time and to be able to offer good genetic counseling to the parents, since the prognosis is not favorable.
\end{abstract}

Keywords: limb - body wall complex, congenital anomaly, exencephaly, gastroschisis, clubfoot, abdominal wall defects

\author{
Volume 7 Issue 6 - 202 I \\ Luis Gustavo Celis,' Isabel Cristina \\ Almonacid, ${ }^{2}$ Esperanza Velásquez, ${ }^{2}$ Isabel \\ Fernández, ${ }^{3}$ José Dorado, ' Andreina Zannin \\ Ferrero,' Paola Zuleta Quintero,' María \\ Camila Garzón Viasus,' Sergio Vergara \\ Cárdenas,' Ana Isabel Bracho Fernandez ${ }^{3}$ \\ 'Universidad de la Sabana - Facultad de Medicina, Colombia \\ ${ }^{2}$ Clínica San Pedro Claver - Departamento de Patología, \\ Colombia \\ ${ }^{3}$ Policlinica Metropolitana,Venezuela
} Correspondence: Andreina Zannin Ferrero, Universidad de la
Sabana - Facultad de Medicina, Colombia, Email inazanninf@gmail.com

Received:September 28, 202I | Published: December 14, 2021

\section{Introduction}

Limb - Body - Wall - Complex is a congenital anomaly of unknown etiology, characterized by craniofacial, thoracoabdominal, spine and lower extremity malformations. This pathology is part of the anomalies related to the abdominal wall, being one of the least frequent. In the present article we present the clinical case of a male neonate with Limb-Body-Wall Complex Syndrome, with a complete clinical history, clinical findings, associated images and a discussion of the available literature on this rare anomaly.

\section{Materials and methods}

\section{Ethical statement, consent and permissions}

This study was conducted under the guidelines of the Declaration of Helsinki, as well as resolution 8430 of 1993 and 2378 of 2008 of the Ministry of Health and Social Protection of Colombia, which safeguard the interests of the subject studied and good clinical practices are adopted.

\section{Subject}

We present the case of a male newborn, who had a complete clinical history and postmortem examination, observing the phenotypic characteristics, in whom blood samples were taken for complementary studies and imaging for the development of this case report.

\section{Methodology}

After the parents had given their informed consent verbally, we proceeded to take the clinical history and post mortem examination of a male newborn, paying special attention to the phenotypic characteristics observed, who was referred for studies by the Department of Pathology for blood sampling, imaging and genetic tests. Subsequently, a literature search was performed in databases such as PubMed, Clinicalkey, Science Direct and OMIM to present the clinical cases reported and the available updated information.

\section{Case report}

\section{Clinical case}

Male newborn, product of first gestation, preterm of a 39 year old mother and 42 year old father, both with blood group $\mathrm{O}$, $\mathrm{RH}+$. Mother with low prenatal controls, with positive results for IgG Toxoplasmosis and Rubeola, negative for HIV. After the born, peripheral blood karyotyping was performed, reporting $46 \mathrm{XY}$ [20]. Normal male karyotype. (Figure 1) to discard chromosomal cause. Born by cesarean section, weight of 2,500 grams, height of $44 \mathrm{~cm}$ and head circumference of $35 \mathrm{~cm}$. On physical examination, multiple malformations were observed such as microphthalmia, micrognathia, low-set ear lobes, short neck (Figure 1), bilateral equinus varus foot, short legs (Figure 2) and gastroschisis in the peritoneal bag showing the liver and intestinal segments (Figure 3). A spine x-ray was performed showing dorsal lumbar cyphoscoliosis, possible hip dysplasia, thorax with decreased anteroposterior diameter and pulmonary hypoplasia (Figure 4\&5).

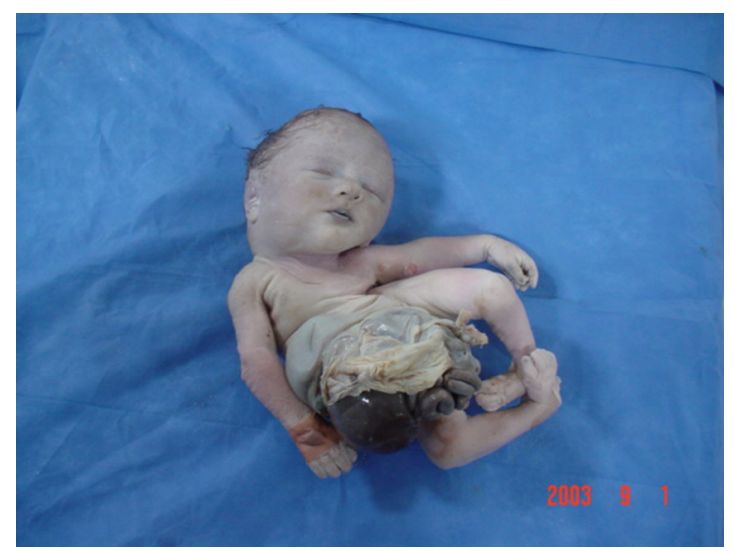

Figure I Male newborn with the characteristics indicated for the Limb - Body Wall Complex. 


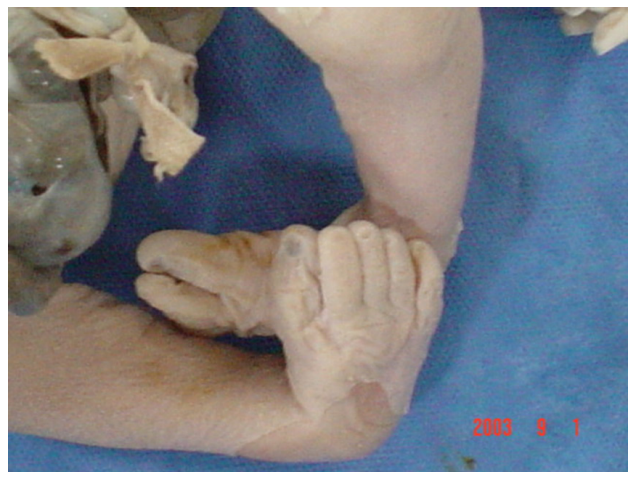

Figure 2 Equinus foot - bilateral varus.

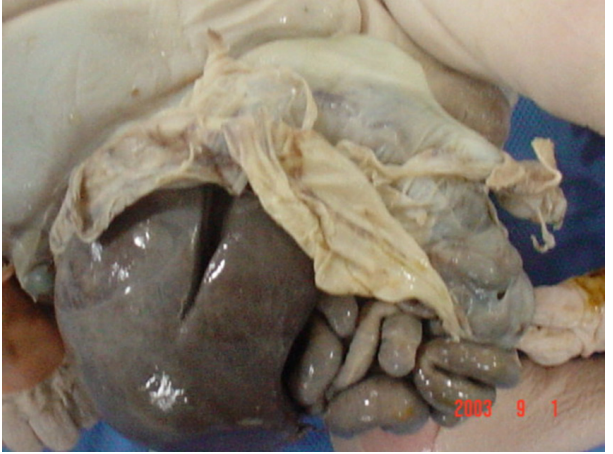

Figure 3 Gastroschisis in the peritoneal pouch showing the liver and intestinal segments.

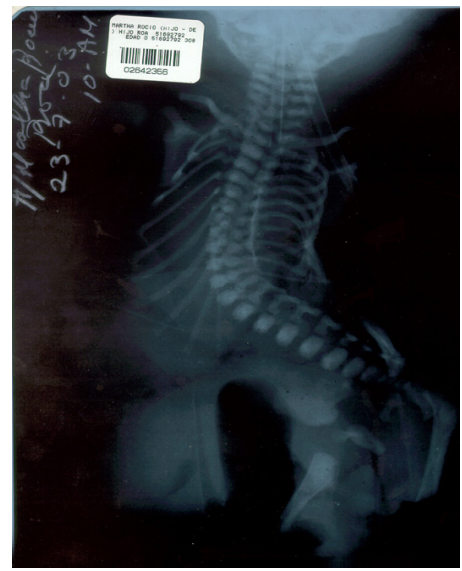

Figure 4 Spine X-ray: dorsal lumbar cyphoscoliosis, hip dysplasia, hypoplasia of the thorax and hypoplasia of both lungs.

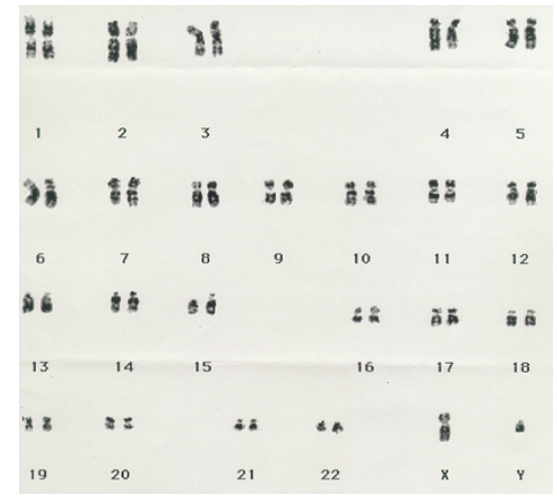

Figure 5 Patient's karyotype 46 XY [20]. Normal male karyotype. No structural or numerical alteration is evident.

\section{Discussion}

The wall - limb complex also known as the Limb Body - Wall Complex or Body Stalk, is a congenital, rare and sporadic anomaly characterized by presenting multiple malformations at the craniofacial and thoracoabdominal level as well as compromising the spine and lower extremities. ${ }^{4}$ Its etiology is not known with certainty; ${ }^{2}$ However, it is known that it develops secondary to an alteration in the formation of the umbilical cord, as it is excessively short (which can occur in up to $96 \%$ of cases), ${ }^{1}$ causing intracavitary organs to be exposed and attached to the placenta and this to the mother's uterus. ${ }^{5}$

There are multiple theories that discuss the origin of this pathology being the most accurate 1) early rupture of the amnion causing a mechanical injury, related to craniofacial and/or limb deformities, 2) vascular disruption during embryogenesis and 3) defective development of the germinal disc. However, the pattern of malformation of this complex varies from the fourth to the sixth week of embryological development ${ }^{2,8}$ according to the fold involved, whether cephalic, caudal or lateral, ${ }^{4}$ generating that there is not a complete development of the abdominal wall. ${ }^{7}$

This pathology is part of the congenital malformations that compromise the abdominal wall, being one of the least frequent in relation to gastroschisis or omphalocele ${ }^{8}$ and with an equal distribution in both sexes. ${ }^{1}$ It can be found in 1 of 14000 to 42000 pregnancies and the incidence ranges from $0.21-0.31 / 10000$ newborns. ${ }^{2}$ Since it is considered a lethal anomaly, it is recommended to perform the interruption and premature termination of pregnancy either naturally or voluntarily at the time of diagnosis, since postnatal survival is unusual, regardless of the fact that recurrence in a subsequent pregnancy is very low. ${ }^{1,3,5}$

However, since its etiology is unknown, experimental studies have been carried out to find causes that imply the obstruction of the development and closure of the abdominal wall, finding a high relationship with the transcription factor AP 2- alpha. It is known that there is a wall compromise that shows total or partial absence of a portion of the wall, including skin, muscle and peritoneum. ${ }^{1}$

According to Van Allen, in 1987 he established the clinical manifestations that could be found taking into account that two of the following three must be present: a) exencephaly/encephalocele and/or facial clefts; b) thoraco and/or abdominoschisis and c) limb defects. ${ }^{3,6}$ There are also two phenotypic categories in which this complex can be classified as defined by Russo, being the cranioplacental, where craniofacial defects and/or upper limb defects can be observed; and the abdominal placental where defects are related to various degrees of thoracoabdominal evisceration, urogenital anomalies, imperforate anus, meningocele, severe spinal deformities, as resembled in our case by observing severe kyphoscoliosis (Figure 4) and involvement of lower extremities such as equinus varus foot (Figure 2). However, it is important to mention that there are cases in which the defects are variable and with different phenotype $\mathrm{e}^{4,7}$ or internal anomalies are highly related (up to $95 \%)^{1}$ such as absence of diaphragm, intestinal atresia or cardiac defects. ${ }^{5}$

Prenatal diagnosis of the wall-limb complex can be made by performing a routine obstetric ultrasound (nuchal translucency ultrasound) in the first trimester of gestation ${ }^{4}$ or when a defect of the ventral wall of the abdominal wall is suspected, ${ }^{1}$ where the fusion of the fetal body including part of the thorax, abdomen and/or pelvis to the placenta will be evidenced..$^{5}$ Abnormalities of the spine and medulla may also be seen. ${ }^{1}$ In the ultrasound image the eviscerated organs will be seen as a complex mass entangled with membranes and 
a large eccentric lateral defect related to the insertion of the umbilical cord. ${ }^{1,8}$ It is important to mention that there are two circumstances in which an adequate ultrasound visualization could be difficult; in case the fetus presents oligohydramnios or if it has any herniated viscera. ${ }^{1}$

In this case the characteristics indicated for Limb-Body Wall Complex anomaly are fulfilled, because the results are compatible with this syndrome. It is important to highlight the poor prognosis of this pathology and the high mortality rate in these patients since in Colombia according to the National Institute of Health in 2017 it was reported that congenital malformations represented $95 \%$ of all reported cases, ${ }^{9}$ so it is of great importance to make an early prenatal diagnosis in order to provide good genetic counseling and calculation of recurrence risk.

\section{Acknowledgments}

None.

\section{Conflicts of interest}

Authors declare no conflict of interest.

\section{Funding}

None.

\section{References}

1. Olaya M, Gómez AM. Limb wall complex at the hospital escuela san ignacio de bogota, colombia:A case report and review of the literature. Revista Colombiana de Obstetricia y Ginecología. 2011;62(4):331-337.

2. Díaz C, Copado Y, Muñoz G, et al. Malformations of the abdominal wall. Revista Médica Clinica Las Condes. 2016; 27(4):499-508.

3. Plakkal N, John J, Jacob S, et al. Limb body wall complex in a still born fetus:a case report. Cases Journal. 2008;1(1):86.

4. Jain JA, Fuchs KM. Limb-body wall complex. In Obstetric Imaging: Fetal Diagnosis and Care, 2nd ed. Elsevier Inc. 2017.

5. Rodriguez A. Anterior trunk wall defects. Ultrasonography In Prenatal Diagnosis. 2008;233-245.

6. Kumtepe Y, Börekç B, Ngeç M, et al. Prenatally Diagnosed Limb Body Wall Complex. 2003;4(69):69-70.

7. Pakdaman R, Woodward PJ, Kennedy A. Complex abdominal wall defects:appearances on prenatal imaging. Radiographics. 2015;35(2):636649.

8. Hagen-ansert SL. Fetal anterior abdominal wall. in textbook of diagnostic sonography. 8th ed. Elsevier Inc. Chapter 62. 2020.

9. National Institute of Health. Congenital defects event report. 2017. 